\title{
Survey of the Huntington's Disease Patient and Caregiver Community Reveals Most Impactful Symptoms and Treatment Needs
}

\author{
Jennifer A. Simpson ${ }^{\mathrm{a}}$, Debra Lovecky ${ }^{\mathrm{a}}$, Jane Kogan ${ }^{\mathrm{b}}$, Louise A. Vetter ${ }^{\mathrm{a}}$ and George J. Yohrling ${ }^{\mathrm{a}, *}$ \\ ${ }^{a}$ Huntington's Disease Society of America (HDSA), New York, NY, USA \\ ${ }^{\mathrm{b}}$ American Board of Perianesthesia Nursing Certification, Inc., New York, NY, USA
}

\begin{abstract}
.
Background: In preparation for a meeting with the U.S. Food and Drug Administration (FDA) on Patient-Focused Drug Development in Huntington's disease, the Huntington's Disease Society of America (HDSA) created and distributed two comprehensive surveys on the symptom experience and treatment approaches for Huntington's disease.

Objective: The objective of these surveys was to identify the specific symptoms that most impact the daily lives of individuals with Huntington's disease/Juvenile Huntington's disease (HD/JHD) and their caregivers and to solicit input on the types of treatments desired by HD affected families. The data were shared with the FDA to offer background and insight in preparation for the patient-focused meeting, as well as to ensure representation by the community in a manner that would complement those who attended in person.

Methods: Two distinct surveys were created using SurveyMonkey to capture patient and caregiver perspectives on HD symptoms and current treatments. HDSA distributed the surveys to the HD community in August and September 2014 and collected responses through January 2015.

Results: More than 3,600 responses to the two surveys were received. The data showed that both caregivers and individuals with HD were severely impacted by the cognitive and behavioral symptoms of HD with HD patients reporting problems with executive functioning and cognitive decline as most impactful to them. However, 30 percent of caregivers reported that chorea was the most impactful symptom compared to 17 percent of people with HD. Across all the symptom categories, patients reported a lower occurrence of symptoms than were reported by their caregivers.

Conclusions: With only one drug approved for treatment of a symptom of Huntington's disease and no disease modifying treatments available, there is a critical need for new medicines to treat the cognitive, psychiatric and motor symptoms associated with HD. While the surveys did not capture risk/benefit data, the data collected do provide new insights around the different perspectives of patients and caregivers. We believe that industry development of treatments would be well-informed by incorporating the patient community, which is more knowledgeable and engaged than given credit, in consideration of treatment regimens, risk-benefit and priorities for therapeutic development.
\end{abstract}

Keywords: Huntington's disease, symptoms, treatments, cognition, chorea, dysexecutive syndrome, Food and Drug Administration, patient-reported outcomes, caregivers, psychiatric

\footnotetext{
${ }^{*}$ Correspondence to: Dr. George Yohrling, Senior Director Mission and Scientific Affairs, HDSA, 505 Eighth Avenue, Suite 902, New York, NY 10018, USA. Tel.: +1 212242 1968; Fax: +1 212 239 3430; E-mail: gyohrling@hdsa.org.
}

\section{INTRODUCTION}

In 2012, the U.S. Food and Drug Administration (FDA) launched a Patient-Focused Drug Development initiative as required under the fifth 
authorization of the Prescription Drug User Fee Act (PDUFA V). The goal of this program was to more systematically gather patients' perspectives on their condition and the available therapies to treat their condition. As part of the process, the FDA committed to obtain the patient and caregiver perspective, focusing on the impact of disease on patients' daily lives, the types of treatment benefit that matters most to patients, and patients' perspectives on the adequacy of available therapies [1]. To fulfill their commitment to the patient community, FDA scheduled approximately 20 public meetings over the duration of PDUFA V, each focused on a specific disease area. HD was selected as one of the disease groups in 2013.

As noted by the FDA, physicians may prescribe a number medications to help control emotional and movement problems associated with HD, but while they may help keep some clinical symptoms under control, there is no current treatment to stop or reverse the course of the disease [2]. In the context of the hearings, the FDA specifically sought information from the patient community that would inform the clinical development and FDA review of potential treatments for HD.

The questions posed to the HD patient and caregiver community from the FDA focused on two topic areas. Topic one encompassed disease symptoms and daily impacts that matter most to patients. Topic two solicited patient perspectives on current approaches to treating HD.

After announcing that the meeting would be held in 2015, the FDA and the Huntington's Disease Society of America (HDSA) discussed the format and above topic areas in order to prepare the community for this important forum. HDSA, founded in 1968 by Marjorie Guthrie, the wife of legendary folk singer Woody Guthrie, is the largest not-for-profit organization dedicated to improving the lives of everyone affected by HD. Through support of clinical Centers of Excellence, research, support groups and social workers, diverse educational programs and advocacy efforts, HDSA aims to provide HD families with "help for today and hope for tomorrow". As part of an outreach and awareness raising campaign to alert the HD community to the FDA's meeting on HD, HDSA distributed two surveys encompassing questions on topic one and topic two and soliciting specific comment on symptomatology, quality of life and therapeutic needs. The community responded en masse, with 2,591 people providing answers for questions in the symptoms survey, and 1,040 people providing answers to questions in the treatments survey.
The approximately 600 patients that responded to the 2 surveys represent $2 \%$ of the estimated 30,000 symptomatic HD patients thought to live in the US.

Prior to these surveys, we are unaware if others had collected data from the HD community on their experiences of symptom impact on daily life or on treatment effectiveness to determine patient-reported outcomes.

Huntington's disease has long been classified as a movement disorder, though prodromal features encompass cognitive and psychiatric symptoms of HD [3]. In response to questions asking caregivers what were the most impactful symptoms of $\mathrm{HD}$, a majority of responses included the cognitive and psychiatric symptoms of the disease. For patients with HD/JHD and their caregivers, dysexecutive syndrome, depression/apathy and chorea were reported as the three most impactful symptoms, in varying order for each group. Dysexecutive syndrome, common in neurologic diseases like $\mathrm{HD}$, is an impairment in cognitive, behavioral, or emotional domains that make executive functions such as planning, cognitive flexibility, initiation and sequencing difficult [4]. The importance of cognitive and behavioral symptoms was clear in the responses of both caregivers and people with HD/JHD. While the prevalence and frequency of symptoms varied between caregivers and people with HD/JHD, the impact of dysexecutive syndrome was obvious for both groups. In addition, the survey on treatment approaches to HD, provided clear evidence to support how limited the availability of efficacious treatments are for people with HD.

We believe that engaging a patient population as early as possible in the clinical development pathway is a critical step to help ensure more efficient clinical trials that will result in more meaningful, effective therapies for the intended patient population.

\section{METHODS}

HDSA used SurveyMonkey, a major provider of web-based surveys, to create two distinct surveys based on discussions with the U.S. Food and Drug Administration (FDA) pertaining to the topics to be covered in the Patient-Focused Drug Development meeting on HD that was to be held September 22, 2015. The first survey, entitled "FDA Topic 1: Disease Symptoms that Matter Most to Individuals with HD", was distributed in August 2014 to members of the HD community and remained open through December 2014. The survey contained 38 questions, divided 
Table 1

Symptom and treatment survey respondent demographics. Average age of caregivers completing surveys was 52.5 years. Average age of individuals with HD/JHD completing surveys was 47.1 years

\begin{tabular}{lcc}
\hline Respondent groups & Survey 1: symptom experience & Survey 2: treatment options \\
\hline Caregivers of someone with HD & 1904 & 801 \\
Caregivers of someone with JHD & 109 & 36 \\
Individual with HD & 536 & 202 \\
Individual with JHD & 20 & 1 \\
\hline
\end{tabular}

into two identical sections, one for individuals with HD/JHD and another for individuals who are or were caregivers for a person with HD. The second survey, entitled "FDA Topic 2: Perspectives on Current Approaches to Treatment", was distributed at the beginning of September 2014 and remained open to collect responses until January 2015. The survey consisted of 167 questions, divided between questions to gather demographic information, and two identical sections of 58 questions to gather data sets from individuals with $\mathrm{HD} / \mathrm{JHD}$ and caregivers of individuals with HD/JHD. The scope of the treatment survey was limited to medication interventions only. It did not inquire about ancillary treatments such as physical, occupational or speech therapy.

The surveys were distributed digitally through multiple HDSA communication channels including social media and HDSA web platforms, in order to reach the greatest number of individuals possible. Respondents could skip questions or choose not to provide information for certain questions on both surveys. As a result, the response rates to questions varied across both surveys. The surveys can be found in the Supplementary data.

\section{RESULTS}

\section{Participation}

Of the 2,591 respondents to survey one on symptoms and their impact on daily life, 1,270 were current caregivers for someone with HD, 634 were caregivers of someone who had HD and is now deceased, 536 were people with HD, 82 were caregivers for someone with JHD, 27 were caregivers for someone who had JHD but is now deceased, and 20 were people with JHD. The average age of caregivers that participated in the surveys was 52.5 years, while the average age of individuals with HD/JHD that participated was 47.1 years.

For survey two, the breakdown of respondents was similar, with more than $75 \%$ of those providing data on treatment options and efficacy being current caregivers of someone with HD or a previous caregiver of a person with HD who has passed away. Similar to survey one, a much smaller percentage of people with HD provided or were able to provide responses compared to caregivers. Only $19.4 \%$ of respondents were people with HD. Only 36 respondents in survey two were current or previous caregivers of someone with JHD, with 1 person with JHD providing a response (Table 1).

\section{Symptoms}

A total of 2,591 people provided responses for the survey related to HD symptoms. Analysis of the data revealed clear similarities in symptom experiences between individuals with HD/JHD and caregivers. However, there were notable differences in how patients and caregivers experienced the negative aspects of HD symptoms. 30\% of HD caregivers most frequently identified chorea as the most impactful symptom on everyday life (Table 2) while $17.5 \%$ of individuals with HD/JHD reported chorea as the most impactful symptom. Instead, individuals with HD/JHD perceived symptoms related to dysexecutive syndrome to be most impactful (22\% of responses). $21.2 \%$ of individuals with $\mathrm{HD} / \mathrm{JHD}$ reported that their inability to work and memory symptoms were most impactful, while $0 \%$ of caregivers reported these symptoms as most impactful for them (Table 2). While caregivers reported chorea as the most impactful individual symptom, 54\% of caregivers also identified a combination of behavioral and cognitive symptoms such as anger/rage, anxiety, cognitive decline and depression/apathy as having great impact.

Caregivers responded more frequently that their loved one with HD had completely lost an ability to perform a task, with $60 \%$ responding that their loved one had completely lost the ability to work, drive, manage finances, take care of family, multi-task, or engage in a sex life. More than $85 \%$ of caregivers responded that their loved one had completely lost the ability to be employed, while only $47 \%$ of individuals 
Table 2

HD Patient and Caregiver perceptions of most impactful symptom of Huntington's disease. HD patients $(\mathrm{N}=189)$, Caregivers $(\mathrm{N}=701)$

\begin{tabular}{lcc}
\hline Symptom & $\begin{array}{c}(\%) \mathrm{HD} \\
\text { Patients }\end{array}$ & (\%) Caregivers \\
\hline Anger/Rage & 10.6 & 9.56 \\
Anxiety & 2.65 & 6.28 \\
Chorea movement/Balance & 17.5 & 30.1 \\
Depression/Apathy & 19.1 & 14.6 \\
Executive function/Cognitive decline & 22.2 & 24.3 \\
Sleep problems & 4.76 & 3.28 \\
Speech and swallowing issues & 4.23 & 14.6 \\
Inability to work & 10.6 & 0.57 \\
Pain & 3.70 & 0.00 \\
\hline
\end{tabular}

with HD/JHD responded similarly. Less than $50 \%$ of HD/JHD respondents said that they had completely lost the ability to do any of the 19 tasks listed in the survey. However, data for both caregivers and individuals with HD/JHD suggest that an inability to maintain employment was among the most frequently experienced effect of HD on their lives (Table 3A).

The perception of overall HD symptom frequency varied greatly between individuals with HD/JHD and caregivers (Fig. 1). Nearly $14 \%$ of the 248 individuals with HD/JHD reported that they never experience HD symptoms, while just $1 \%$ of caregivers suggested that they never observe symptoms in their loved ones. Greater than $80 \%$ of caregivers said they saw symptoms in their loved one constantly, while less than $35 \%$ of individuals with HD/JHD reported constant HD symptoms.

Caregivers reported that they observed behavioral symptoms such as delusions and hallucinations in individuals with HD/JHD approximately $228 \%$ more frequently than did individuals with $\mathrm{HD} / \mathrm{JHD}$ (Table 3B). These results support a previous study that demonstrated that individuals with HD show increasing unawareness of frontally-mediated behaviors as the disease progressed [5]. Delusions and hallucinations are rarely seen in early stage HD. The large difference in symptom perception between patient and caregiver could be simply because the HD patients responding to this survey are likely in the early stages of HD while the caregivers are reporting on all stages of HD. Both caregiver and patients reported depression at similar rates $(71 \%$ and $79 \%$ respectively).

Caregivers reported cognitive symptoms in their loved one at greater rates compared to individuals with $\mathrm{HD} / \mathrm{JHD}$. At least $50 \%$ of caregivers reported their loved one experienced 14 of the 16 cognitive symptoms listed for HD, while individuals with
HD/JHD reported experiencing 6 of the 16 cognitive symptoms $50 \%$ of the time (Table 3C). The cognitive symptom with the largest perceived difference between caregiver and patient was unawareness. Caregivers reported unawareness of symptoms $76 \%$ more often than patients.

The responses of caregivers and individuals with HD/JHD aligned more closely in the prevalence of motor symptoms. Both groups reported that gait impairment/falls was the most prevalently experienced motor symptom of HD (Supplemental data 1).

\section{Treatments}

A total of 1,040 people provided responses for the survey related to HD treatments. Data showed that very few individuals were taking any medications, prescriptive or non-prescriptive, for symptoms associated with dysexecutive syndrome. More than $80 \%$ of respondents noted that they or their loved one with HD were not taking any kind of medication for deterioration in memory and thinking. Similarly, more than $40 \%$ of respondents noted that they or their loved one were not utilizing any form of medication to manage perseveration or anxiety, which can also be associated with dysexecutive syndrome. Respondents listed 10 different medication options for anxiety and depression with sertraline (Zoloft) being the most prescribed (Supplemental data). Approximately $80 \%$ of caregivers believed existing drugs were at least somewhat effective in managing anxiety or perseveration.

For behavioral symptoms like depression, there are effective treatments available. Respondents noted 11 different medications being used to treat depression, with sertraline being used most often to treat these symptoms (Table 4). Just $15.8 \%$ of respondents noted that depression medications managed the symptom "not very well" or "not at all" (Fig. 2). For other symptoms that caregivers and HD patients reported as having a major impact, such as anger/rage and irritability, nearly $40 \%$ of respondents reported not taking any medication to treat these symptoms. Nineteen different medication options were reported as possible treatments for the anger and irritability seen in HD. The majority of respondents reported that medications worked "somewhat" to manage this symptom. For other behavioral symptoms like apathy and unawareness, there were no reports of effective treatments.

The only FDA approved drug to treat a symptom of HD is tetrabenazine (Xenazine), which is used to treat chorea associated with HD. Even with 
Table 3

A) HD patient and caregiver reported loss of ability to perform ADLs HD Patients $(\mathrm{N}=238)$ Caregivers $(\mathrm{N}=735)$. \% Difference $=(\%$ caregivers-\% HD patients)/\% HD Patients * 100. B) HD Patient and Caregiver reported experience of behavioral symptoms. HD Patients $(\mathrm{N}=216)$, Caregivers $(\mathrm{N}=720) . \%$ Difference $=(\%$ caregivers-\% HD patients $) / \%$ HD Patients $* 100 . \mathrm{C})$ HD patient and caregiver reported experience of cognitive symptoms of HD. HD Patients $(\mathrm{N}=215)$, Caregivers $(\mathrm{N}=729)$

\begin{tabular}{|c|c|c|c|c|}
\hline \multirow[t]{20}{*}{$\mathrm{A}$} & Activity of Daily Living (ADL) & (\%) HD Patients & (\%) Caregivers & (\%) Difference \\
\hline & Participating in Family Events & 2.55 & 22.0 & 764.7 \\
\hline & Physical Activities & 3.54 & 29.5 & 733.9 \\
\hline & Taking care of Family & 8.23 & 65.1 & 690.5 \\
\hline & Ability to concentrate & 4.39 & 28.5 & 548.5 \\
\hline & Household chores/errands & 9.13 & 54.0 & 491.2 \\
\hline & Relations with others & 5.13 & 26.9 & 424.8 \\
\hline & Planning Activities & 11.4 & 52.9 & 365.9 \\
\hline & Relations with family & 3.07 & 13.4 & 335.2 \\
\hline & Ability to manage finances & 16.3 & 70.4 & 331.8 \\
\hline & Sex life & 14.6 & 62.3 & 326.4 \\
\hline & Ability to multitask & 15.7 & 63.4 & 305.4 \\
\hline & Ability to remember & 3.91 & 14.9 & 281.8 \\
\hline & Enjoyment of life & 5.60 & 21.2 & 278.4 \\
\hline & Emotional well-being & 6.11 & 21.2 & 247.5 \\
\hline & Driving & 24.7 & 75.7 & 208.8 \\
\hline & Falling asleep at night & 7.33 & 13.5 & 83.49 \\
\hline & Employment & 47.8 & 85.8 & 79.24 \\
\hline & Sleeping during the day & 5.73 & 9.62 & 67.89 \\
\hline & Sleeping at night & 12.6 & 19.9 & 58.25 \\
\hline \multirow[t]{12}{*}{$\mathrm{B}$} & Behavioral Symptom & (\%) HD Patients & (\%) Caregivers & (\%) Difference \\
\hline & Delusions and Hallucinations & 7.41 & 24.3 & 228.1 \\
\hline & Mania & 12.0 & 22.5 & 86.88 \\
\hline & Obsessions and Compulsions & 37.0 & 67.2 & 81.48 \\
\hline & Demoralization & 18.5 & 30.8 & 66.47 \\
\hline & Apathy & 46.3 & 55.0 & 18.76 \\
\hline & Sleep Problems & 66.2 & 72.1 & 8.880 \\
\hline & Irritability & 69.0 & 69.4 & 0.670 \\
\hline & Sexual Problems & 35.7 & 34.4 & -3.390 \\
\hline & Anxiety & 76.9 & 70.1 & -8.730 \\
\hline & Depression & 79.2 & 70.7 & -10.71 \\
\hline & Panic Disorder & 34.3 & 28.6 & -16.49 \\
\hline \multirow[t]{17}{*}{$\overline{\mathrm{C}}$} & Cognitive Symptom & (\%) HD Patients & $(\%)$ Caregivers & (\%) Difference \\
\hline & Unawareness & 35.4 & 62.41 & 76.55 \\
\hline & Impaired initiation of speech & 46.1 & 73.80 & 60.26 \\
\hline & Perseveration & 42.3 & 65.67 & 55.14 \\
\hline & Difficulty with emotional recognition & 40.9 & 60.22 & 47.13 \\
\hline & Difficulty with perception of time & 43.3 & 61.59 & 42.37 \\
\hline & Difficulty with spatial perception & 36.3 & 48.87 & 34.70 \\
\hline & Impulsivity & 48.4 & 61.73 & 27.59 \\
\hline & Dysexecutive syndrome & 67.0 & 85.46 & 27.58 \\
\hline & Disorganization of language content & 41.4 & 51.99 & 25.58 \\
\hline & Decline in cognitive processing speed & 69.3 & 86.83 & 25.30 \\
\hline & Learning problems & 48.4 & 59.26 & 22.51 \\
\hline & Decline in attention & 65.6 & 76.54 & 16.71 \\
\hline & Lack of initiation & 61.9 & 71.06 & 14.87 \\
\hline & Implicit memory loss & 51.2 & 58.44 & 14.23 \\
\hline & Irritability/Temper outbursts & 70.7 & 68.45 & -3.18 \\
\hline & Difficulty with smell identification & 20.0 & 18.38 & -8.10 \\
\hline
\end{tabular}

an FDA approved treatment, nearly $40 \%$ of respondents reported being unaware of or not utilizing any medication to treat chorea. Just $22.9 \%$ of respondents noted taking tetrabenazine to treat chorea, while
$15.8 \%$ used risperidone (Table 5). Nearly $25 \%$ of respondents said that existing chorea medications work "not very well" or "not at all" to suppress the symptom (Fig. 3). 
Frequency of HD Symptom Experience

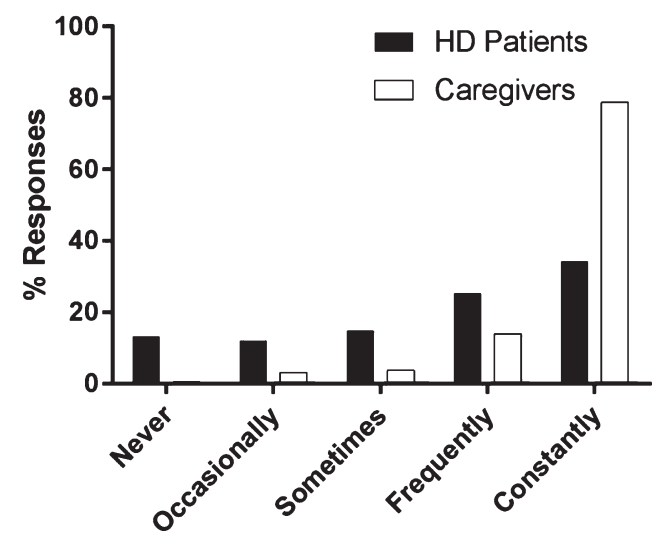

Fig. 1. HD patient and caregiver perspectives on frequency of HD symptom experience. HD patient $(\mathrm{N}=248)$, HD caregiver $(\mathrm{N}=731)$.

Table 4

Reported treatment options for Depression in Huntington's disease $(\mathrm{N}=255)$

\begin{tabular}{lc}
\hline Treatment & $\%$ \\
\hline No Treatment & 34.5 \\
Sertraline & 16.9 \\
Citalopram & 11.0 \\
Buproprion & 7.84 \\
I Don't Know & 7.84 \\
Fluoxetine & 7.06 \\
Escitalopram & 5.49 \\
Venlafaxine & 5.49 \\
Paroxetine & 5.10 \\
Valproic Acid & 4.31 \\
Duloxetine & 2.75 \\
Desvenlafaxine & 1.57 \\
Lamotrigine & 1.18 \\
\hline
\end{tabular}

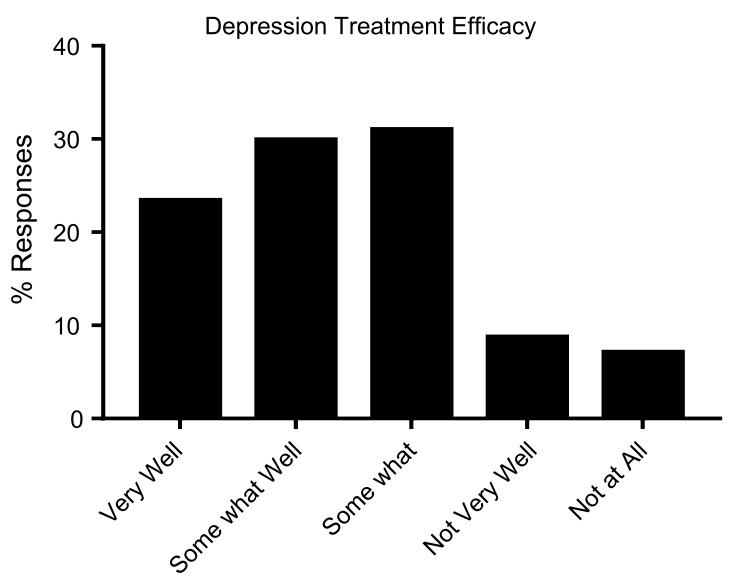

Fig. 2. Reported efficacy of available treatment for depression in HD patients $(\mathrm{N}=184)$.
Table 5

Reported treatment options for chorea in Huntington's disease $(\mathrm{N}=310)$

\begin{tabular}{lc}
\hline Treatment & $\%$ \\
\hline No Treatment & 25.2 \\
Tetrabenazine & 22.9 \\
Risperidone & 15.8 \\
Clonazepam & 13.9 \\
Lorazepam & 12.6 \\
Haloperidol & 10.7 \\
Olanzapine & 10.0 \\
Not Applicable & 9.35 \\
Quetiapine & 9.35 \\
I don't know & 4.84 \\
Amantadine & 3.55 \\
Memantine & 2.90 \\
Aripiprazole & 2.58 \\
Ziprasidone & 1.29 \\
Fluphenazine & 0.00 \\
\hline
\end{tabular}

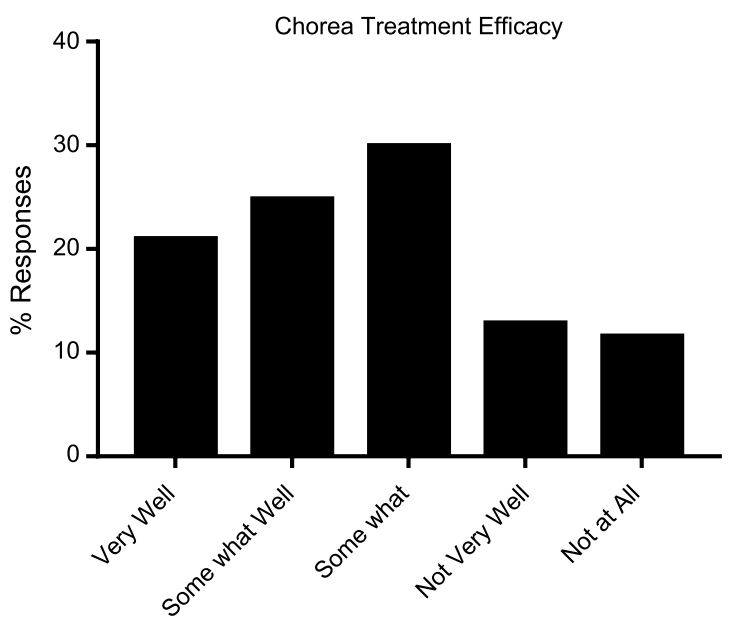

Fig. 3. Reported efficacy of available treatments for chorea in HD patients $(\mathrm{N}=234)$.

\section{DISCUSSION}

The voice of the HD patient and caregiver can be an invaluable resource in helping to determine directions for drug development, clinical trials and approvals. Although it is a newer tool, the usefulness of the direct insight into the patient experience of a condition can provide a more comprehensive perspective of the benefits and risks from potential treatments [7]. Patient reported outcomes (PRO) have been utilized previously as supplementary evidence for approval of anti-cancer products, including directly measuring the benefits of a treatment on the ability to function and symptom experience [6]. 
What is striking in these data are the marked differences in the experience and perception of symptoms of HD and their impact between individuals with HD/JHD and their caregivers. The frequency and impact of symptoms appears to be felt more by caregivers than individuals with $\mathrm{HD}$, especially when it comes to loss of abilities. There was also a large difference in the perception of symptom frequency between caregivers and individuals with HD. More than $90 \%$ of caregivers surveyed responded that their loved one experienced HD symptoms "frequently" or "constantly" while only $50 \%$ of individuals with HD responded similarly. Caregivers also perceived their loved one with HD as much more impaired than the individual themselves. This may be attributed to anosognosia (unawareness) that has previously been reported in HD and other neurological disorders [7-8]. Approximately $67 \%$ of caregivers responded that unawareness made it harder for their loved one to manage symptoms that were treatable with medication (Supplementary data 2). If the patient does not believe they exhibit depression or chorea, they may be less inclined to take medications to help with these symptoms.

With that being said, it is important to note how individuals with $\mathrm{HD} / \mathrm{JHD}$ responded when asked to reflect on their symptom experiences. The most impactful symptoms of HD were related to their ability to maintain independence. Individuals with HD/JHD reported loss of ability to maintain employment, perform household chores and drive more frequently compared to abilities that did not impact independence as directly, such as the ability to concentrate or multi-task. Interestingly, sleep irregularities, which are a common early feature in HD were not regarded by patients nor caregivers as being that impactful on their lives [9].

The loss of independence can be linked back to the reported symptoms experienced by individuals with HD and caregivers, including cognitive and behavioral aspects of HD. Symptoms, such as slowed cognitive processing speed, decline in attention, dysexecutive syndrome and irritability/anger outbursts all affect an individual's ability to work, to drive and to manage a household. These symptoms affect the individual with HD even in the pre-manifest stage, with an increasing prevalence of irritability, apathy and executive dysfunction as the disease progresses [10]. The importance of finding effective treatments is clear from both the perspective of the individual with HD and the caregiver, with both groups feeling the impact of these symptoms.
Survey respondents noted that there were few to no treatments for the cognitive symptoms of HD, like memory loss or decline in cognitive processing and thinking, even though these symptoms were the most impactful for individuals with HD.

Similarly, for behavioral symptoms like irritability and anger, apathy, obsessions and compulsions and sleep problems, there remains a lack of effective treatment options. Behavioral symptoms were frequently cited by respondents as having a negative impact on the lives of individuals with HD and their caregivers. For caregivers, there was not a single behavioral symptom, but rather a combination of symptoms associated with HD that together were more impactful than the motor symptoms. There was also no clear cut best practice treatment option for symptoms like irritability, apathy, anxiety or depression, as demonstrated by the numerous medications respondents noted using to treat these symptoms with varying rates of effectiveness. The large degree of heterogeneity in HD behavioral symptoms makes them difficult to be included as a reliable endpoint in a clinical trial. More quantitative and objective behavioral clinical readouts for HD must be developed so that novel drugs targeting the behavioral components can be properly evaluated in HD clinical trials.

Although there is an FDA approved treatment for chorea (tetrabenazine), there are no effective treatments for symptoms like gait impairment/falls, speech impairment, or swallow impairment/choking. Even with the treatments available for chorea, the majority of respondents reported that treatments were only "somewhat" effective in managing symptoms. Since symptoms like chorea and gait impairments/falls were reported as some of the most commonly experienced motor symptoms of HD, there is still a critical need for improvement and diversity in the treatment options for the motor symptoms of HD.

The efforts of the FDA in the implementation of the patient-focused drug development meetings and other forums to solicit the patient perspective are laudable and are already creating a positive impact on the lives of patients across the spectrum of diseases. Patient views from the melanoma community regarding the psychological and cosmetic benefits to seeing a melanoma lesion shrink that would not normally respond to conventional and previously approved therapies were influential in the FDA's decision to grant approval for Imlygic (Talimogene laherparepvec). Perhaps the most high profile result of patient feedback on the drug development process 
came in September 2016 when Dr. Janet Woodcock, the Director of the Center for Drug Evaluation and Research (CDER) at the FDA overruled an advisory panel that voted 7-6 against approval Sarepta Therapeutic's Exondys 51 (eteplirsen) for the treatment of Duchenne muscular dystrophy (DMD). Some believe that vocal disease advocates may have influenced this decision despite a lack of clinical efficacy data [11].

While the insight into the patient and caregiver perspective we have gained from these surveys is important, there are limitations to this methodology. First, the patients and caregivers responding are not matched dyads, meaning not all patients that responded had a caregiver who also participated. We also have no way of confirming the diagnosis of anyone responding as an "HD patient". Given these surveys were completed online, the patient perspective is likely biased towards the earlier stage HD patients that were physically able to participate. Gaining a true understanding of late stage HD patients will require caregiver feedback.

One important aspect not covered in this FDA process and the corresponding surveys to the community was understanding the risks HD patients are willing to take for effective therapies to treat each of the different symptoms a patient experiences. Future studies should query HD patients to delineate the risks they are willing to take to gain access to an experimental therapy. While one may not hesitate to be subjected to regular intra-striatal injections for a potential disease-modifying therapy, being subjected to similar injections to ameliorate the chorea associated with HD or other less impactful symptoms, would likely be met with less enthusiasm. This could translate into a poorly recruited/delayed clinical trial.

In summary, patient and caregiver perspectives on HD symptomatology and treatment efficacy should be critical components in shaping future HD clinical development efforts. With this information we can tailor efforts to treat the symptoms that those impacted by HD state are the most disruptive more effectively and target symptoms that degrade quality of life. While there seems to be some effective treatments for symptoms like depression and anxiety, there are still cognitive, behavioral and motor symptoms which lack effective treatments and are severely impacting the lives of both individuals with HD and their caregivers. Taking this information into account, we can refocus efforts in research and development to offer more options, and hopefully more effective treatments for individuals impacted by Huntington's disease.

\section{ACKNOWLEDGMENTS}

The Huntington's Disease Society of America would like to thank the thousands of individuals with HD/JHD and families that took the time to provide their experiences so that HDSA could hold a meaningful conversation with the FDA and pharmaceutical and biotech partners as they continue the path of clinical development for new and effective therapies to treat HD.

\section{CONFLICT OF INTEREST}

There are no conflicts to report related to the surveys, their collection or analysis.

\section{SUPPLEMENTARY MATERIAL}

Supplementary Data 1: HD Patient and Caregiver reports on experience of motor symptoms of HD. Supplementary Data 2: Caregiver perspective on impact of unawareness in making it more difficult to manage HD symptoms treatable with medication. Supplementary data 3: Surveys distributed to HD community regarding symptoms and treatments.

The supplementary material is available in the electronic version of this article: http://dx.doi.org/ 10.3233/JHD-160228.

\section{REFERENCES}

[1] U.S. Food and Drug Administration. The Voice of the Patient: A series of reports from the U.S. Food and Drug Administration's (FDA's) Patient-Focused Drug Development Initiative. U.S. Food and Drug Administration; 2016 p. 3.

[2] U.S. Food and Drug Administration. Public Meeting on Patient-Focused Drug Development for Huntington's and Parkinson's Diseases [Internet]. 2015. Available from: https://www.regulations.gov/document?D=FDA-2012-N0967-0747

[3] Stout JC, Paulsen JS, Queller S, Solomon AC, Whitlock $\mathrm{KB}$, Campbell JC, et al. Neurocognitive signs in prodromal Huntington disease. Neuropsychology. 2011;25(1):1-14.

[4] Hanna-Pladdy B. Dysexecutive syndromes in neurologic disease. J Neurol Phys Ther. 2007;31(3):119-27.

[5] Hergert D, Sanchez-Ramos J, Cimino C. Examining Huntington's disease patient and informant concordance on frontally mediated behaviors. J Clin Exp Neuropsychol. 2015;37(9):981-7.

[6] Turner-Bowker D, Hao Y, Foley C, Galipeau N, Mazar I, Krohe M, et al. The use of patient-reported outcomes in advanced breast cancer clinical trials: A review of the published literature. Curr Med Res Opin [Internet]. 2016 [cited 9 August 2016]; Available from: http://www. tandfonline.com/doi/abs/10.1080/03007995.2016.1205005 
[7] Hocaoglu M, Gaffan E, Ho A. The Huntington's disease health-related Quality of Life questionnaire (HDQoL): A disease-specific measure of health-related quality of life. Clin Genet. 2012;81(2):117-22.

[8] Kaptein A, Scharloo M, Helder DI, Snoei L, van Kempen GMJ, Weinman J, et al. Quality of life in couples living with Huntington's disease: The role of patients' and partners' illness perceptions. Qual Life Res. 2007;16(5):793-801.

[9] Goodman AO, Rogers L, Pilsworth S, McAllister CJ, Shneerson JM, Morton AJ, et al. Asymptomatic sleep abnormalities are a common early feature in patients with Huntington's disease. Curr Neurol Neurosci Rep. 2011;11(2):211-7.

[10] Sheppard D, Pirogovsky-Turk E, Woods S, Holden H, Nicoll D, Filoteo JV, et al. Everyday Functioning in Huntington's disease: A laboratory-based study of financial management capacity. Appl Neuropsychol Adult. 2016:1-7.

[11] Railroading at the FDA. [Editorial]. Nat Biotechnol. 2016;34(11):1078. 